\title{
Liste der Berufskrankheiten erweitert, Meldung auch bei Verdacht!
}

— Die in aktualisierter Fassung von der Bundesanstalt für Arbeitsschutz und Arbeitsmedizin veröffentlichte „Liste der Berufskrankheiten“ wurde unter Berücksichtigung neuer medizinischer For- schungsergebnisse um fünf Krankheiten erweitert: Erkrankungen des Blutes durch Benzol, Gonarthrose durch kniende Tätigkeiten, Lungenkrebs durch polyzyklische aromatische Kohlenwasserstoffe, Lun-

\section{MMW Kommenta}

Die ärztliche Meldepflicht ist im SGB VII gesetzlich verankert. Hat ein Arzt den begründeten Verdacht, dass bei einem Patienten eine Berufskrankheit vorliegt, ist er verpflichtet, dies dem Träger der Unfallversicherung oder den für den medizinischen Arbeitsschutz zuständigen Stellen unverzüglich anzuzeigen ( $\$ 202$ SGB VII). Der Patient ist über den Inhalt der Anzeige zu unterrichten. Unfallversicherungsträger oder die staatliche Arbeitsschutzstelle, der die Anzeige zugeleitet wird, müssen ihm mitgeteilt werden.

Die Verpflichtung zur Meldung durch den Arzt ist allerdings weder von einer Einwilligung des Patienten abhängig, noch hat dieser ein Widerspruchsrecht. Nach den Vorstellungen des Gesetzgebers soll eine Anzeigepflicht auch dann bestehen, wenn der Versicherte eine solche Anzeige nicht wünscht. Darüber hinaus ermöglicht eine Öffnungsklausel im Absatz 2 des \$9 SGB VII die Anerkennung von Berufskrankheiten, die unter keiner Position der aktuellen Berufskrankheitenliste aufgeführt sind, sofern neue wissenschaftliche Erkenntnisse vorliegen, die zum Zeitpunkt der Verabschiedung der Liste noch nicht bekannt oder geprüft worden sind. Die Meldung einer Berufskrankheit erfolgt auf dem Vordruck $F 6000$ und kann nach der Nr. 141 UV-GOÄ (15,22 Euro) gegenüber der zuständigen Unfallversicherung in Rechnung gestellt werden. genkrebs durch das Zusammenwirken von Asbestfaserstaub und polyzyklischen aromatischen Kohlenwasserstoffen sowie Lungenfibrose durch Schweißrauche und -gase.

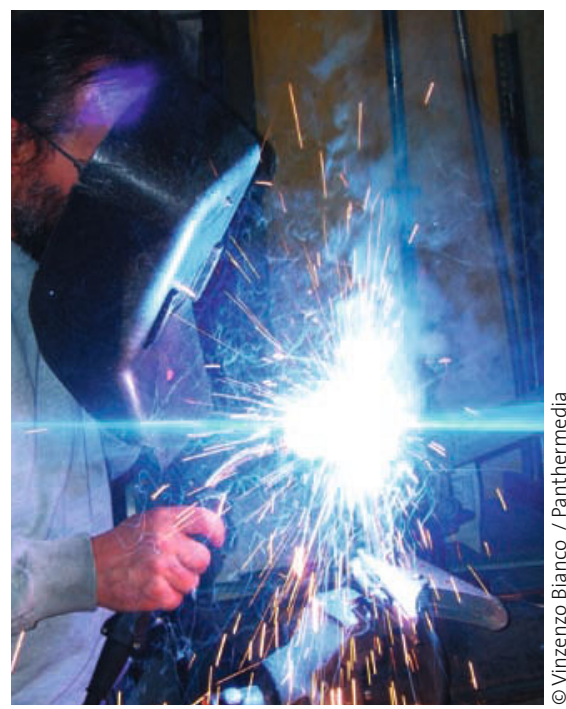

Lungenfibrose bei Schweißern ist jetzt als Berufskrankheit anerkannt.

\section{Glitazone ausgeschlossen, Glinide können noch verordnet werden}

- Der vom Gemeinsamen Bundesausschuss (G-BA) beschlossene Verordnungsausschluss zu den Glitazonen ist am 18.11.2010 im Bundesanzeiger erschienen und gilt damit ab dem Beginn des übernächsten Quartals. Das wäre der 1. April 2011. Rosiglitazon wurde bereits vom Hersteller vom Markt genommen.

Im Hinblick auf den Verordnungsausschluss der Glinide hat das Bundesgesundheitsministerium (BMG) hingegen den G-BA zu einer weiteren Stellungnahme aufgefordert. Nach Auffassung des Ministeriums müssen noch hinreichende
Belege vorgelegt werden, dass die Verordnung von Gliniden unter Berücksichtigung der neuen Rechtslage des AMNOG tatsächlich unzweckmäßig sei.

\section{MMW Kommentar}

Beachtenswert ist, dass der Verordnungsausschluss für Glitazone sowohl für Mono- wie auch Kombinationspräparate gilt. Das BMG hebt in seinem Schreiben vor allem auf wirtschaftliche Aspekte ab. Der $G-B A$ soll angeben, mit welchen Einsparungen die Kassen durch den Ausschluss der Glinide rechnen können. Der G-BA sei auf diese Frage in seiner ersten Antwort nicht eingegangen. Daneben bezieht sich das BMG auf mögliche Folgen des geplanten Ausschlusses. Der G-BA hatte kritisiert, dass zu den Gliniden keine Langzeitstudien mit patientenrelevanten Endpunkten vorlägen. Gleichzeitig gebe es mit Metformin und den Sulfonylharnstoffen Substanzen, deren Nutzen erwiesen sei. Das BMG weist nun darauf hin, dass diese Wirkstoffe wegen möglicher Kontraindikationen und Unverträglichkeiten nicht bei allen Diabetikern eingesetzt werden könnten. 